\title{
On Two Definitions of Reduct ${ }^{\star}$
}

\author{
Manuel S. Lazo-Cortés, José Fco. Martínez-Trinidad, \\ and J.A. Carrasco-Ochoa \\ Instituto Nacional de Astrofísica, Óptica y Electrónica \\ Mexico \\ $\{\mathrm{mlazo}$, fmartine, ariel\}@ccc.inaoep. $\mathrm{mx}$
}

\begin{abstract}
This paper studies the relationship between the two most common definitions of reduct. Although there are other definitions, almost all the literature published in the framework of the Theory of Rough Sets, uses one of the two definitions we study here. However, there is an ambiguity in the use of these definitions and often authors do not previously declare what definition they refer to. Moreover, there are no publications where the relation between these two definitions is widely discussed, just that is what this paper addresses. We enunciate and demonstrate several properties expressing relations between both definitions including some illustrative examples.
\end{abstract}

\section{Introduction}

Object classification is normally accompanied and usually preceded by a task of feature selection, which reduces the dimension of the space of representation of objects. Intensive research on feature selection and pattern discovery has given us several new representations and approaches that use different formalisms. In the framework of the Rough Set Theory [7], the basic concept for the mentioned aim is the concept of reduct for a decision table. Informally, the concept of reduct, refers to a subset of attributes that retains the same ability to discern between object pairs in a table as the full set of attributes do.

In the literature, there are several definitions of reduct, see for example $3-8$, 11]. Nevertheless, reducts, in any definition, combine two properties, the capacity of preserving certain property of the set of all attributes (normally associated to differentiating power) and being of minimal cardinality among all subsets fulfilling this property. It means that attributes in a reduct are jointly sufficient and individually necessary to differentiate among object descriptions.

We decided to examine the relation between the two definitions more commonly used in the literature.

This document is organized as follows. Section 2 provides the formal background for the study of both definitions of reduct. In this section, we include

\footnotetext{
* This work was partly supported by the National Council of Science and Technology of Mexico (CONACyT) through the project grants CB2008-106443 and CB2008106366.
} 
several examples for supporting our discussion. Section 3 contains a list of theoretical relations between both types of reducts, including a case study. Our remarks are summarized in Section 4.

\section{Basic Concepts}

In many data analysis applications, information and knowledge are stored and represented as an information table because this information table provides a convenient way to describe a finite set of objects within a universe through a finite set of attributes [7]. Reduct is a key concept within the Rough Set Theory, defined for information systems or decision tables.

Definition 1. (information system) An information system is a pair $\mathcal{S}=\left(U, A_{t}^{*}\right)$ where $U$ is a finite non-empty set of objects, $A_{t}^{*}$ is a finite non-empty set of conditional (descriptive) attributes. Each $a \in A_{t}^{*}$ corresponds to the function $I_{a}$ : $U \rightarrow V_{a}$ called evaluation function, where $V_{a}$ is called the value set of a.

An information table is the simplest form of an information system. It can be represented as a matrix, in which rows are associated to objects, columns to attributes and cells to values of attributes on objects. Without loss of generality, in this paper, we focus our discussion on a type of information table called decision table.

Definition 2. (decision table) A special type of information table is denoted as $\mathcal{S}_{d}=\left(U, A_{t}=A_{t}^{*} \cup\{d\}\right)$, where $A_{t}^{*}$ is a set of conditional attributes and $d$ is a decision attribute $(\{d\}=D)$ indicating the decision class for each object in the universe. Such information table is called a decision table.

When considering decision tables, it is important to distinguish between the so called consistent and the inconsistent ones. A decision table is said to be consistent, if each combination of values of descriptive attributes uniquely determines the value of the decision attribute, and inconsistent, otherwise. Later we will define these concepts formally.

For introducing the concept of reduct, the indiscernibility relation plays a special role.

Definition 3. (indiscernibility relation) Given a subset of conditional attributes $A \subseteq A_{t}^{*}$, the indiscernibility relation $I N D(A) \subseteq U \times U$ for an information system is defined as

$$
I N D(A)=\left\{(u, v) \in U \times U: \forall a \in A ; I_{a}(u)=I_{a}(v)\right\}
$$

For a decision table $\mathcal{S}_{d}$, we can define the relative indiscernibility relation as

$$
I N D(A \mid D)=\left\{(u, v) \in U \times U: \forall a \in A,\left[I_{a}(u)=I_{a}(v)\right] \vee\left[I_{d}(u)=I_{d}(v)\right]\right\}
$$


In practice, it is quite common that decision tables contain descriptions of a finite sample $\mathcal{U}$ of objects from a larger (possibly infinite) universe $U$, where values of descriptive attributes are always known for all objects from $U$, but decision is in general a hidden function except for those objects from the sample $\mathcal{U}$. The main problem of learning theory is to generalize the decision function (defined on the sample $\mathcal{U}$ ) to the whole universe $U$. In this case, we formally have a decision table $\mathcal{S}_{d}=\left(\mathcal{U}, A_{t}=A_{t}^{*} \cup\{d\}\right)$ and an information table $\mathcal{S}=$ $\left(U, A_{t}^{*}\right)$, being $\mathcal{U} \subseteq U$. The decision attribute allows partitioning the universe into blocks determined by all possible decisions.

Definition 4. (decision class) For $k \in V_{d}$, a decision class is defined as $U_{k}=$ $\left\{u \in U: I_{d}(u)=k\right\}$. Let us denote the cardinality of $U_{k}$ by $m_{k} ;$ so $\left|U_{k}\right|=m_{k}$. Based on this definition, we can write $U / I N D(D)=\left\{U_{1}, U_{2}, \ldots, U_{\left|V_{d}\right|}\right\}$.

Based on the relative indiscernibility relation, a reduct [8] is defined as follows.

Definition 5. (reduct for a decision table) Given a decision table $\mathcal{S}_{d}$, an attribute set $R \subseteq A_{t}^{*}$ is called a reduct, if $R$ satisfies the following two conditions:

(i) $I N D(R \mid D)=I N D\left(A_{t}^{*} \mid D\right)$ (if $R$ satisfies (i) it is called a super reduct);

(ii) For any $a \in R, I N D((R-\{a\}) \mid D) \neq I N D\left(A_{t}^{*} \mid D\right)$.

Other definition widely used requires that a certain region of the universe be preserved, this region is called positive region. Next, we will introduce it.

Definition 6. (lower and upper approximations) Let $A \subseteq A_{t}$ be a subset of attributes and let $[u]_{A}$ be the block (class) of the partition $U \mid I N D(A)$ containing $u \in U$. We define the A-lower approximation of a set $X \subseteq U$ as follows:

$$
\underline{A}(X)=\left\{u \in U:[u]_{A} \subseteq X\right\}
$$

The A-upper approximation of $X$ is defined as

$$
\bar{A}(X)=\left\{u \in U:[u]_{A} \cap X \neq \emptyset\right\}
$$

The set $\bar{A}(X)-\underline{A}(X)$ is called the boundary region of $X$.

The notion of rough set is just associated to the boundary region.

Definition 7. (rough set) $X$ is a rough set if it has a non-empty boundary region (i.e. $\bar{A}(X)-\underline{A}(X) \neq \emptyset)$. Otherwise $X$ is a crisp set.

Definition 8. (positive region) Given a decision table $\mathcal{S}_{d}=\left(U, A_{t}=A_{t}^{*} \cup\{d\}\right)$ and a subset of attributes $A \subseteq A_{t}^{*}$. The $A$-positive region with respect to $d$ is defined as

$$
P O S_{\{d\}}(A)=\bigcup_{X \in U / I N D(\{d\})} \underline{A}(X)
$$

We say that a decision table $\mathcal{S}_{d}=\left(U, A_{t}=A_{t}^{*} \cup\{d\}\right)$ is deterministic or consistent if $\operatorname{POS}_{\{d\}}\left(A_{t}^{*}\right)=U$. Otherwise, we call it non-deterministic or inconsistent. Another commonly used definition of reduct is [9]: 
Definition 9. (relative reduct) Given a decision table $\mathcal{S}_{d}=\left(U, A_{t}=A_{t}^{*} \cup\{d\}\right)$, an attribute subset $R \subseteq A_{t}^{*}$ is called a relative reduct, if $R$ satisfies the two conditions:

(i) $\operatorname{POS}_{\{d\}}(R)=P O S_{\{d\}}\left(A_{t}^{*}\right)$; (if $R$ satisfies (i) is called a relative super reduct)

(ii) $\operatorname{POS}_{\{d\}}(R-\{a\}) \neq P O S_{\{d\}}\left(A_{t}^{*}\right)$ for any $a \in R$.

As an initial step in understanding the relationship between the two definitions of reduct, let analyze the following example.

Example 1. Let us consider the decision table in Table 1 where $U=\left\{u_{1}, u_{2}, u_{3}\right.$, $\left.u_{4}, u_{5}, u_{6}, u_{7}\right\}, A_{t}^{*}=\left\{a_{1}, a_{2}, a_{3}, a_{4}\right\}$ and $D=\{d\}$. Notice that Table 1 is inconsistent, see for example that $I_{\left\{a_{1}, a_{2}, a_{3}, a_{4}\right\}}\left(u_{2}\right)=I_{\left\{a_{1}, a_{2}, a_{3}, a_{4}\right\}}\left(u_{6}\right)=(0,0,0,0)$ but $0=I_{d}\left(u_{2}\right) \neq I_{d}\left(u_{6}\right)=1$.

We have that

$I N D\left(A_{t}^{*} \mid D\right)=\left\{\left(u_{1}, u_{1}\right),\left(u_{2}, u_{2}\right),\left(u_{3}, u_{3}\right),\left(u_{4}, u_{4}\right),\left(u_{5}, u_{5}\right),\left(u_{6}, u_{6}\right),\left(u_{7}, u_{7}\right)\right.$, $\left\langle u_{1}, u_{2}\right\rangle,\left\langle u_{1}, u_{3}\right\rangle,\left\langle u_{1}, u_{4}\right\rangle,\left\langle u_{2}, u_{3}\right\rangle,\left\langle u_{2}, u_{4}\right\rangle,\left\langle u_{3}, u_{4}\right\rangle,\left\langle u_{5}, u_{6}\right\rangle,\left\langle u_{5}, u_{7}\right\rangle,\left\langle u_{6}, u_{7}\right\rangle$, $\left.\left\langle u_{2}, u_{6}\right\rangle,\left\langle u_{3}, u_{6}\right\rangle,\left\langle u_{4}, u_{5}\right\rangle\right\}$, where $\left\langle u_{i}, u_{j}\right\rangle$ denotes the two elements $\left(u_{i}, u_{j}\right)$ and $\left(u_{j}, u_{i}\right)$.

Let us consider the attribute set $R=\left\{a_{1}, a_{2}, a_{3}\right\}$, then

$I N D(R)=\left\{\left(u_{1}, u_{1}\right),\left(u_{2}, u_{2}\right),\left(u_{3}, u_{3}\right),\left(u_{4}, u_{4}\right),\left(u_{5}, u_{5}\right),\left(u_{6}, u_{6}\right),\left(u_{7}, u_{7}\right)\right.$, $\left.\left\langle u_{2}, u_{3}\right\rangle,\left\langle u_{2}, u_{6}\right\rangle,\left\langle u_{3}, u_{6}\right\rangle,\left\langle u_{4}, u_{5}\right\rangle\right\}=I N D\left(A_{t}^{*}\right)$,

therefore $I N D(R \mid D)=I N D\left(A_{t}^{*} \mid D\right)$.

Following definition [5, $R$ is a super reduct for this decision table.

Let analyze if $R$ is a reduct, and for this purpose let consider the subsets $R_{1}$ $=R-\left\{a_{1}\right\}=\left\{a_{2}, a_{3}\right\}, R_{2}=R-\left\{a_{2}\right\}=\left\{a_{1}, a_{3}\right\}$ and $R_{3}=R-\left\{a_{3}\right\}=\left\{a_{1}, a_{2}\right\}$, for which we have that

$I N D\left(R_{1} \mid D\right)=I N D\left(A_{t}^{*} \mid D\right) \cup\left\{\left\langle u_{2}, u_{5}\right\rangle,\left\langle u_{3}, u_{5}\right\rangle,\left\langle u_{4}, u_{6}\right\rangle\right\} ; \operatorname{IND}\left(R_{2} \mid D\right)=$ $I N D\left(A_{t}^{*} \mid D\right) \cup\left\{\left\langle u_{1}, u_{7}\right\rangle\right\}$ and $I N D\left(R_{3} \mid D\right)=I N D\left(A_{t}^{*} \mid D\right) \cup\left\{\left\langle u_{1}, u_{6}\right\rangle\right\}$.

Table 1. An inconsistent decision table

\begin{tabular}{ccccccc}
\hline$U$ & $a_{1}$ & $a_{2}$ & $a_{3}$ & $a_{4}$ & $d$ \\
\hline \hline$u_{1}$ & 0 & 0 & 1 & 1 & 0 \\
$u_{2}$ & 0 & 0 & 0 & 0 & 0 \\
$u_{3}$ & 0 & 0 & 0 & 0 & 0 \\
$u_{4}$ & 1 & 0 & 0 & 0 & 0 \\
$u_{5}$ & 1 & 0 & 0 & 0 & 1 \\
$u_{6}$ & 0 & 0 & 0 & 0 & 1 \\
$u_{7}$ & 0 & 1 & 1 & 0 & 1
\end{tabular}

According to condition (ii) in definition 5, we can say that $R$ is a reduct for this decision table.

Now, let us consider the definition 9, we need to calculate $P O S_{\{d\}}(R)$; then 
$U / I N D(\{d\})=\left\{\left\{u_{1}, u_{2}, u_{3}, u_{4}\right\},\left\{u_{5}, u_{6}, u_{7}\right\}\right\}$

$U / I N D\left(A_{t}^{*}\right)=\left\{\left\{u_{1}\right\},\left\{u_{2}, u_{3}, u_{6}\right\},\left\{u_{4}, u_{5}\right\},\left\{u_{7}\right\}\right\}$ and

$\underline{A}_{t}^{*}\left(\left\{u_{1}, u_{2}, u_{3}, u_{4}\right\}\right)=\left\{u_{1}\right\}=\underline{R}\left(\left\{u_{1}, u_{2}, u_{3}, u_{4}\right\}\right)$;

$\underline{A}_{t}^{*}\left(\left\{u_{5}, u_{6}, u_{7}\right\}\right)=\left\{u_{7}\right\}=\underline{R}\left(\left\{u_{5}, u_{6}, u_{7}\right\}\right)$,

and therefore $P O S_{\{d\}}\left(A_{t}^{*}\right)=\left\{u_{1}, u_{7}\right\}=P O S_{\{d\}}(R)$;

so $R$ satisfies condition (i) of definition 9 and we can say that $R$ is a relative super reduct.

Let analyze condition (ii):

$U / I N D\left(R_{1}\right)=\left\{\left\{u_{1}\right\},\left\{u_{2}, u_{3}, u_{4}, u_{5}, u_{6}\right\},\left\{u_{7}\right\}\right\} ;$

$U / I N D\left(R_{2}\right)=\left\{\left\{u_{1}, u_{7}\right\},\left\{u_{2}, u_{3}, u_{6}\right\},\left\{u_{4}, u_{5}\right\}\right\}$ and

$U / I N D\left(R_{3}\right)=\left\{\left\{u_{1}, u_{2}, u_{3}, u_{6}\right\},\left\{u_{4}, u_{5}\right\},\left\{u_{7}\right\}\right\}$; then

$\underline{R}_{1}\left(\left\{u_{1}, u_{2}, u_{3}, u_{4}\right\}\right)=\left\{u_{1}\right\}$,

$\underline{R}_{2}\left(\left\{u_{1}, u_{2}, u_{3}, u_{4}\right\}\right)=\emptyset$ and

$\underline{R}_{3}\left(\left\{u_{1}, u_{2}, u_{3}, u_{4}\right\}\right)=\emptyset$ and

$\underline{R}_{1}\left(\left\{u_{5}, u_{6}, u_{7}\right\}\right)=\left\{u_{7}\right\}$,

$\underline{R}_{2}\left(\left\{u_{5}, u_{6}, u_{7}\right\}\right)=\emptyset$ and

$\underline{R}_{3}\left(\left\{u_{5}, u_{6}, u_{7}\right\}\right)=\left\{u_{7}\right\}$

thence $\operatorname{POS}_{\{d\}}\left(R-\left\{a_{1}\right\}\right)=\operatorname{POS}_{\{d\}}\left(A_{t}^{*}\right)$. It means that $R$ does not fulfill condition $(i i)$ of definition 9 . We can conclude that $R$ is a reduct of the decision table in Table 1 according to definition [5, but it is not a reduct if we use definition 9 .

It should be emphasized that although in some publications the equivalence between both definitions of reduct here included (5) and 9) is handled lightly; in 9] it is accurately established (Proposition 5.6, page 351) that this equivalence holds if $I N D(A)=I N D(\{d\})$. Meanwhile, Bazan and Szczuka [2] also remark that in the presence of an inconsistent table the notion of generalized decision, which is defined below, has to be used.

Definition 10. (generalized decision) Let $\mathcal{S}_{d}=\left(U, A_{t}=A_{t}^{*} \cup\{d\}\right)$ be a decision table, the generalized decision function $\partial$ is defined as $\partial(u)=\left\{k \in V_{d}: \exists v \in\right.$ $\left.U\left[(u, v) \in I N D\left(A_{t}^{*}\right) \wedge I_{d}(v)=k\right]\right\}$. Any set consisting of all objects with the same generalized decision value is called a generalized decision class.

Now, we can say that $\mathcal{S}_{d}$ is consistent or deterministic if $|\partial(u)|=1$ for any $u \in U$. An important consequence of the last definition is that it is possible to transform an arbitrary inconsistent decision table $\mathcal{S}_{d}=\left(U, A_{t}=A_{t}^{*} \cup\{d\}\right)$ into a consistent decision table $\mathcal{S}_{\partial}=\left(U, A_{t}^{*} \cup\{\partial\}\right)$.

We will differentiate with names and notations between the reducts defined by definition 5 (we will call them discerning decision reducts and we will denote the set of all these reducts of a decision table $\mathcal{S}_{d}$ by $R E D_{\text {ind }}\left(\mathcal{S}_{d}\right)$ ) and those defined by definition 9 (which we will call positive region decision reducts, and we will denote the set of all positive region decision reducts of a decision table $\mathcal{S}_{d}$ by $\left.R E D_{\text {pos }}\left(\mathcal{S}_{d}\right)\right)$, analogously we will denote as $D S_{\text {ind }}\left(\mathcal{S}_{d}\right)$ and $D S_{\text {pos }}\left(S_{d}\right)$ the set of super reducts determined by definitions 5 and 9 respectively. Sometimes, to point out that the generalized decision is being used, we will use the notation $\mathcal{S}_{\partial}$ instead of $\mathcal{S}_{d}$. 


\section{Relations}

In this section, we present the theoretical relations between both types of reducts. Since $\mathcal{S}_{\partial}$ is a consistent table, first we can derive the following fact.

Lemma 1. Let $\mathcal{S}_{d}=\left(U, A_{t}=A_{t}^{*} \cup\{d\}\right)$ a decision table, then $R E D_{\text {pos }}\left(\mathcal{S}_{d}\right)=$ $R E D_{\text {ind }}\left(\mathcal{S}_{\partial}\right)=R E D_{\text {pos }}\left(\mathcal{S}_{\partial}\right)$.

The following theorem establishes a relation between the set of discerning decision super reducts and the set of positive region decision super reducts of a decision table.

Theorem 1. Let $\mathcal{S}_{d}=\left(U, A_{t}=A_{t}^{*} \cup\{d\}\right)$ a decision table, then $D S_{\text {ind }}\left(\mathcal{S}_{d}\right) \subseteq$ $D S_{\text {pos }}\left(\mathcal{S}_{\partial}\right)$. If $\mathcal{S}_{d}$ is consistent the equality holds.

Proof. The implication $\left[I N D(R)=I N D\left(A_{t}^{*}\right)\right] \Rightarrow\left[P O S_{\{d\}}(R)=P O S_{\{d\}}\left(A_{t}^{*}\right)\right]$ naturally follows from definition of relative indiscernibility relation and definitions 8 and [6. From this is immediate that $D S_{\text {ind }}\left(\mathcal{S}_{d}\right) \subseteq D S_{\text {pos }}\left(\mathcal{S}_{d}\right)$.

Now let $\mathcal{S}_{d}$ be a consistent table and let $R \in D S_{\text {pos }}\left(\mathcal{S}_{d}\right)$; it means that $P O S_{\{d\}}(R)=P O S_{\{d\}}\left(A_{t}^{*}\right)=U$.

Let us suppose that $\operatorname{IND}(R \mid D) \neq I N D\left(A_{t}^{*} \mid D\right)$, obviously in this case $I N D\left(A_{t}^{*} \mid D\right) \subset I N D(R \mid D)$. Let $(u, v) \in I N D(R \mid D)-I N D\left(A_{t}^{*} \mid D\right)$; it means that $A_{t}^{*}$ discerns between $u$ and $v$ but $R$ does not. Hence we have that $I_{R}(u)=I_{R}(v)$ and $I_{d}(u) \neq I_{d}(v)$.

Let us denote as $[u]_{\{d\}}$ and $[u]_{R}$ respectively the class to which $u$ belongs in $U / I N D(d)$ and $U / I N D(R)$. We have that $[u]_{\{d\}} \neq[v]_{\{d\}}$ but $[u]_{R}=[v]_{R}$, then $[u]_{R} \nsubseteq[u]_{\{d\}}$ and $[v]_{R} \nsubseteq[v]_{\{d\}}$ and hence $u \notin P O S_{\{d\}}(R)$ and $v \notin P O S_{\{d\}}(R)$ which is a contradiction.

Thus we have that $I N D(R \mid D)=I N D\left(A_{t}^{*} \mid D\right)$ and therefore $R \in D S_{\text {ind }}\left(\mathcal{S}_{d}\right)$ and hence $D S_{\text {ind }}\left(\mathcal{S}_{d}\right)=D S_{\text {pos }}\left(\mathcal{S}_{d}\right)$.

It is important to highlight that sometimes the equality holds even if we do not have consistent tables, as it can be seen from Example 2 .

Example 2. Consider the decision table $\mathcal{S}_{d}$ in Table 2 being $U=\left\{u_{1}, u_{2}, u_{3}\right.$, $\left.u_{4}, u_{5}, u_{6}\right\}, A_{t}^{*}=\left\{a_{1}, a_{2}, a_{3}\right\}$ and $D=\{d\}$. Notice that Table 2 is inconsistent since $I_{\left\{a_{1}, a_{2}, a_{3}\right\}}\left(u_{2}\right)=I_{\left\{a_{1}, a_{2}, a_{3}\right\}}\left(u_{5}\right)=(1,0, \mathrm{H})$ and $0=I_{d}\left(u_{2}\right) \neq I_{d}\left(u_{5}\right)=1$.

Table 2. Another inconsistent table

\begin{tabular}{c||cccc}
\hline$U$ & $a_{1}$ & $a_{2}$ & $a_{3}$ & $d$ \\
\hline \hline$u_{1}$ & 0 & 1 & $\mathrm{H}$ & 0 \\
$u_{2}$ & 1 & 0 & $\mathrm{H}$ & 0 \\
$u_{3}$ & 1 & 1 & $\mathrm{~V}$ & 0 \\
$u_{4}$ & 0 & 1 & $\mathrm{~N}$ & 1 \\
$u_{5}$ & 1 & 0 & $\mathrm{H}$ & 1 \\
$u_{6}$ & 0 & 1 & $\mathrm{~V}$ & 0
\end{tabular}


We have that

$I N D(D)=\left\{\left(u_{1}, u_{1}\right),\left(u_{2}, u_{2}\right),\left(u_{3}, u_{3}\right),\left(u_{4}, u_{4}\right),\left(u_{5}, u_{5}\right),\left(u_{6}, u_{6}\right),\left\langle u_{1}, u_{2}\right\rangle\right.$, $\left.\left\langle u_{1}, u_{3}\right\rangle,\left\langle u_{1}, u_{6}\right\rangle,\left\langle u_{2}, u_{3}\right\rangle,\left\langle u_{2}, u_{6}\right\rangle,\left\langle u_{3}, u_{6}\right\rangle,\left\langle u_{4}, u_{5}\right\rangle\right\}$.

$U / I N D(D)=\left\{\left\{u_{1}, u_{2}, u_{3}, u_{6}\right\},\left\{u_{4}, u_{5}\right\}\right\}$

$\left.I N D\left(A_{t}^{*}\right)=\left\{\left(u_{1}, u_{1}\right),\left(u_{2}, u_{2}\right),\left(u_{3}, u_{3}\right),\left(u_{4}, u_{4}\right),\left(u_{5}, u_{5}\right),\left(u_{6}, u_{6}\right),\left\langle u_{2}, u_{5}\right\rangle\right)\right\}$.

$U / I N D\left(A_{t}^{*}\right)=\left\{\left\{u_{1}\right\},\left\{u_{2}, u_{5}\right\},\left\{u_{3}\right\},\left\{u_{4}\right\},\left\{u_{6}\right\}\right\}$

$I N D\left(A_{t}^{*} \mid D\right)=\left\{\left(u_{1}, u_{1}\right),\left(u_{2}, u_{2}\right),\left(u_{3}, u_{3}\right),\left(u_{4}, u_{4}\right),\left(u_{5}, u_{5}\right),\left(u_{6}, u_{6}\right),\left\langle u_{1}, u_{2}\right\rangle\right.$, $\left.\left\langle u_{1}, u_{3}\right\rangle,\left\langle u_{1}, u_{6}\right\rangle,\left\langle u_{2}, u_{3}\right\rangle ;\left\langle u_{2}, u_{6}\right\rangle,\left\langle u_{3}, u_{6}\right\rangle,\left\langle u_{4}, u_{5}\right\rangle,\left\langle u_{2}, u_{5}\right\rangle\right\}$

Let us consider the subsets $R_{1}=A_{t}^{*}-\left\{a_{1}\right\}=\left\{a_{2}, a_{3}\right\}, R_{2}=A_{t}^{*}-\left\{a_{2}\right\}=$ $\left\{a_{1}, a_{3}\right\}$ and $R_{3}=A_{t}^{*}-\left\{a_{3}\right\}=\left\{a_{1}, a_{2}\right\}$.

We have that

$I N D\left(R_{1} \mid D\right)=I N D\left(R_{2} \mid D\right)=I N D\left(A_{t}^{*} \mid D\right)$ and

$I N D\left(R_{3} \mid D\right)=I N D\left(A_{t}^{*} \mid D\right) \cup\left\{\left\langle u_{1}, u_{4}\right\rangle,\left\langle u_{4}, u_{6}\right\rangle\right\}$.

From this we have that $D S_{\text {ind }}\left(\mathcal{S}_{d}\right)=\left\{R_{1}, R_{2}, A_{t}^{*}\right\}$ and $R E D_{\text {ind }}\left(\mathcal{S}_{d}\right)=$ $\left\{R_{1}, R_{2}\right\}$. Notice that $I N D\left(\left\{a_{3}\right\} \mid D\right)=I N D\left(A_{t}^{*} \mid D\right) \cup\left\{\left\langle 1_{2}, u_{5}\right\rangle\right\}$ therefore $\left\{a_{3}\right\} \notin$ $D S_{\text {ind }}\left(\mathcal{S}_{d}\right)$, being $\left\{a_{1}\right\} \subset R_{3}$ and $\left\{a_{2}\right\} \subset R_{3}$ no singleton in $U$ is a super reduct.

Now, let us calculate the corresponding positive regions

$\underline{A}_{t}^{*}\left(\left\{u_{1}, u_{2}, u_{3}, u_{6}\right\}\right)=\left\{u_{1}, u_{3}, u_{6}\right\}$

$\underline{A}_{t}^{*}\left(\left\{u_{4}, u_{5}\right\}\right)=\left\{u_{4}\right\}$

and therefore $\operatorname{POS}_{\{d\}}\left(A_{t}^{*}\right)=\left\{u_{1}, u_{3}, u_{4}, u_{6}\right\}$

$U / I N D\left(R_{1}\right)=\left\{\left\{u_{1}\right\},\left\{u_{2}, u_{5}\right\},\left\{u_{4}\right\},\left\{u_{3}, u_{6}\right\}\right\}=U / I N D\left(R_{2}\right)$ and

$U / I N D\left(R_{3}\right)=\left\{\left\{u_{1}, u_{4}, u_{6}\right\},\left\{u_{2}, u_{5}\right\},\left\{u_{3}\right\}\right\}$

then $\underline{R}_{1}\left(\left\{u_{1}, u_{2}, u_{3}, u_{6}\right\}\right)=\left\{u_{1}, u_{3}, u_{6}\right\}=\underline{R}_{2}\left(\left\{u_{1}, u_{2}, u_{3}, u_{6}\right\}\right)$

and $\underline{R}_{3}\left(\left\{u_{1}, u_{2}, u_{3}, u_{6}\right\}\right)=\left\{u_{3}\right\}$

$\underline{R}_{1}\left(\left\{u_{4}, u_{5}\right\}\right)=\left\{u_{4}\right\}=\underline{R}_{2}\left(\left\{u_{4}, u_{5}\right\}\right)$ and $\underline{R}_{3}\left(\left\{u_{4}, u_{5}\right\}\right)=\emptyset$;

and thence $P O S_{\{d\}}\left(R_{1}\right)=P O S_{\{d\}}\left(R_{2}\right)=P O S_{\{d\}}\left(A_{t}^{*}\right)=\left\{u_{1}, u_{3}, u_{4}, u_{6}\right\}$ and $P O S_{\{d\}}\left(R_{3}\right)=\left\{u_{3}\right\}$.

Then we have that $D S_{\text {pos }}\left(\mathcal{S}_{d}\right)=\left\{R_{1}, R_{2}, A_{t}^{*}\right\}$ and $R E D_{\text {pos }}\left(\mathcal{S}_{d}\right)=\left\{R_{1}, R_{2}\right\}$. In this case, despite being an inconsistent table, we have that $D S_{\text {ind }}\left(\mathcal{S}_{d}\right)=$ $D S_{\text {pos }}\left(\mathcal{S}_{d}\right)$.

Let $\mathcal{S}_{d}=\left(U, A_{t}^{*} \cup\{d\}\right)$ be a (possibly inconsistent) decision table, and let us introduce the decision attribute $\Delta$ defined as

$$
\Delta(u)=\left\{\begin{array}{l}
{[u]_{\partial} \text { if }[u]_{\partial} \subseteq[u]_{\{d\}}} \\
{[u]_{A_{t}^{*}} \text { otherwise }}
\end{array}\right.
$$

Theorem 2. Let $\mathcal{S}_{d}=\left(U, A_{t}^{*} \cup\{d\}\right)$ be a decision table and let $\mathcal{S}_{\Delta}=\left(U, A_{t}^{*} \cup\right.$ $\{\Delta\})$ being $\Delta$ defined as in (1), then $\mathcal{S}_{\Delta}$ is consistent.

Proof. Let suppose that $\mathcal{S}_{d}$ is consistent, then for every $u \in U$ we have that $[u]_{\partial}=[u]_{\{d\}}$ and hence $[u]_{\Delta}=[u]_{\partial}=[u]_{\{d\}}$. It means that $U / \operatorname{IND}(\{\Delta\})=$ $U / I N D(\{\partial\})=U / I N D(\{d\})$ so $\mathcal{S}_{\Delta}$ is consistent.

Let us now consider that $\mathcal{S}_{d}$ is inconsistent and let $u \in U$ such that $[u]_{\partial} \subseteq$ $[u]_{\{d\}}$; in this case, from (11) we have that $[u]_{\Delta}=[u]_{\partial} \subseteq[u]_{\{d\}}$.

Let suppose now that $[u]_{\partial} \nsubseteq[u]_{\{d\}}$, then from (11) $[u]_{\Delta}=[u]_{A_{t}^{*}}$ and from definition of generalized decision (definition 10 ) we can conclude that $[u]_{A_{t}^{*}} \subseteq$ 
$[u]_{\partial}$; thereby $U / I N D(\{\Delta\})$ is a finer partition than $U / I N D(\{\partial\})$. Therefore $\mathcal{S}_{\Delta}$ is consistent.

From theorem 2, since $S_{\Delta}$ is consistent, it is immediate the following.

Corollary 1. Let $\mathcal{S}_{d}=\left(U, A_{t}^{*} \cup\{d\}\right)$ be a decision table and let $\mathcal{S}_{\Delta}=\left(U, A_{t}^{*} \cup\right.$ $\{\Delta\})$ built as above. Then $R E D_{\text {ind }}\left(\mathcal{S}_{\Delta}\right)=R E D_{\text {pos }}\left(\mathcal{S}_{\Delta}\right)$.

From theorem 2 and from the definitions of $\Delta(u)$ and $\partial(u)$ we also have that:

Corollary 2. Let $\mathcal{S}_{d}=\left(U, A_{t}^{*} \cup\{d\}\right)$ be a decision table and let $\mathcal{S}_{\Delta}=\left(U, A_{t}^{*} \cup\right.$ $\{\Delta\})$ built as above. Then $R E D_{\text {ind }}\left(\mathcal{S}_{d}\right)=R E D_{\text {pos }}\left(\mathcal{S}_{\Delta}\right)$.

The theorems and properties enunciated in this section confirm the nonequivalence between the two definitions of reduct. But even more, our study gives us the possibility of using algorithms to compute one type of reducts in contexts where algorithms to compute other type are used and vice versa. For example Corollary 2 provides a method for computing discerning decision reducts (definition 5) for an inconsistent table by using a software like RSES [2] which was designed for computing positive region decision reducts (definition 9).

\subsection{Case Study}

Here we illustrate the non-equivalence between the two definitions of reduct using the dataset Spect from the UCI Machine Learning Repository [1] .

The Spect dataset consists of two non-disjoint classes, each class containing 40 objects and 22 descriptive attributes, but there are several coincidences in object descriptions. Table 3 shows these coincidences.

Description (I) $a_{1}=a_{2}=a_{3}=a_{4}=a_{5}=a_{6}=a_{7}=a_{8}=a_{9}=a_{10}=a_{11}=$ $a_{12}=a_{13}=a_{14}=a_{15}=a_{16}=a_{17}=a_{18}=a_{19}=a_{20}=a_{21}=a_{22}=0$ appears 12 times in class 0 and twice in class 1 . Obviously none reduct can discern among these objects. Likewise, description (II) $a_{1}=a_{2}=a_{3}=a_{4}=a_{5}=a_{6}=a_{7}=$ $a_{8}=a_{9}=a_{10}=a_{11}=a_{12}=a_{13}=a_{14}=a_{15}=a_{16}=a_{17}=a_{18}=a_{19}=a_{20}$ $=a_{21}=0, a_{22}=1$ appears 3 times in class 0 and once in class 1 . Descriptions (III) $a_{1}=a_{2}=a_{3}=a_{4}=a_{5}=a_{6}=a_{7}=a_{8}=a_{9}=a_{10}=a_{11}=a_{12}=a_{13}=$ $a_{14}=a_{15}=a_{16}=a_{17}=a_{18}=a_{19}=a_{21}=a_{22}=0, a_{20}=1$ and (IV) $a_{1}=1$, $a_{2}=a_{3}=a_{4}=0, a_{5}=1, a_{6}=a_{7}=a_{8}=a_{9}=0, a_{10}=1, a_{11}=a_{12}=a_{13}$ $=a_{14}=a_{15}=a_{16}=a_{17}=a_{18}=0, a_{19}=1, a_{20}=a_{21}=a_{22}=0$ appear once in each class. The last two columns in Table 3 ( T(0) and $\mathrm{T}(1))$ show the times each description appears in class 0 and class 1 respectively.

From the Spect dataset we created two modified datasets: Spect3c and Spect6c. Spect3c results from creating a new class containing the intersection between the two original classes and Spect6c results from separating each repeated description as a new class. Notice that Spect3c is the table resulting from applying generalized decision; in Table 4 we denote it by $\mathcal{S}_{\partial}$, meanwhile Spect6c corresponds to $\mathcal{S}_{\Delta}$.

Table 4 presents the amount of discerning decision reducts (column (A)) and positive region decision reducts $(\operatorname{column}(\mathrm{B}))$ from the original Spect dataset and 
Table 3. Repeated objects in the Spect dataset

\begin{tabular}{ccccccccccccccccccccccccccc}
\hline & $a_{1}$ & $a_{2}$ & $a_{3}$ & $a_{4}$ & $a_{5}$ & $a_{6}$ & $a_{7}$ & $a_{8}$ & $a_{9}$ & $a_{10}$ & $a_{11}$ & $a_{12}$ & $a_{13}$ & $a_{14}$ & $a_{15}$ & $a_{16}$ & $a_{17}$ & $a_{18}$ & $a_{19}$ & $a_{20}$ & $a_{21}$ & $a_{22}$ & $\mathrm{~T}(0)$ & $\mathrm{T}(1)$ \\
\hline \hline (I) & 0 & 0 & 0 & 0 & 0 & 0 & 0 & 0 & 0 & 0 & 0 & 0 & 0 & 0 & 0 & 0 & 0 & 0 & 0 & 0 & 0 & 0 & 12 & 2 \\
(II) & 0 & 0 & 0 & 0 & 0 & 0 & 0 & 0 & 0 & 0 & 0 & 0 & 0 & 0 & 0 & 0 & 0 & 0 & 0 & 0 & 0 & 1 & 3 & 1 \\
(III) & 0 & 0 & 0 & 0 & 0 & 0 & 0 & 0 & 0 & 0 & 0 & 0 & 0 & 0 & 0 & 0 & 0 & 0 & 0 & 1 & 0 & 0 & 1 & 1 \\
(IV) & 1 & 0 & 0 & 0 & 1 & 0 & 0 & 0 & 0 & 1 & 0 & 0 & 0 & 0 & 0 & 0 & 0 & 0 & 1 & 0 & 0 & 0 & 1 & 1 \\
\hline
\end{tabular}

Table 4. Discerning decision reducts and positive region decision reducts for the Spect dataset

\begin{tabular}{lccccc}
\hline Dataset & $\begin{array}{c}\text { Descriptive } \\
\text { attributes }\end{array}$ & Classes & Objects & $\left|R E D_{\text {ind }}\left(S_{*}\right)\right|$ & $\left|R E D_{\text {pos }}\left(S_{*}\right)\right|$ \\
\hline \hline Spect $=\left(\mathcal{S}_{d}\right)$ & 22 & 2 & 80 & 26 & 70 \\
Spect3c $=\mathcal{S}_{\partial}$ & 22 & 3 & 80 & 70 & 70 \\
Spect6c $=\mathcal{S}_{\Delta}$ & 22 & 6 & 80 & 26 & 26 \\
\hline$*\{d, \partial, \Delta\}$. & & & & & \\
\hline$*\{d, \Delta$
\end{tabular}

for each one of the two modified datasets before described. Although Table 4 contains only quantities and not the sets, we can see the equalities $\left|R E D_{\text {ind }}\left(\mathcal{S}_{d}\right)\right|$ $=\left|R E D_{\text {pos }}\left(\mathcal{S}_{\Delta}\right)\right|($ Corollary 2$)$ and $\left|R E D_{\text {pos }}\left(\mathcal{S}_{d}\right)\right|=\left|R E D_{\text {pos }}\left(\mathcal{S}_{\partial}\right)\right|($ Lemma 1$)$. Since $\mathcal{S}_{\partial}$ and $\mathcal{S}_{\Delta}$ are consistent tables, values in columns (A) and (B) in second and third rows show that $\left|R E D_{\text {ind }}\left(\mathcal{S}_{\partial}\right)\right|=\left|R E D_{\text {pos }}\left(\mathcal{S}_{\partial}\right)\right|$ and $\left|R E D_{\text {ind }}\left(\mathcal{S}_{\Delta}\right)\right|=$ $\left|R E D_{\text {pos }}\left(\mathcal{S}_{\Delta}\right)\right|$. For this experiment, reducts were computed by using RSES [2].

\section{Conclusions}

This paper studies the relation between the two most important definitions of reduct demonstrating and illustrating that although in many cases (particularly when classes are disjoint, and tables are consistent) the two concepts coincide, in general they are not the same. As we show in the study case, our study gives us, as an immediate result, the possibility of using algorithms to compute reducts by definition 5 , in contexts where algorithms to compute reducts by definition 9 are used and vice versa. Knowledge of the true relationship between these concepts can make proper use of each approach. This study can be a starting point for further considerations regarding other definitions of reduct.

\section{References}

1. Bache, K., Lichman, M.: UCI Machine Learning Repository. University of California, School of Information and Computer Science, Irvine, CA (2013), http://archive.ics.uci.edu/ml 
2. Bazan, J.G., Szczuka, M.S.: The Rough Set Exploration System. In: Peters, J.F., Skowron, A. (eds.) Transactions on Rough Sets III. LNCS, vol. 3400, pp. 37-56. Springer, Heidelberg (2005)

3. Chen, D.G., Wang, C.Z., Hu, Q.H.: A new approach to attribute reduction of consistent and inconsistent covering decision systems with covering rough sets. Information Sciences 177, 3500-3518 (2007)

4. Kryszkiewicz, M.: Comparative studies of alternative type of knowledge reduction in inconsistent systems. International Journal of Intelligent Systems 16, 105-120 (2001)

5. Mi, J.S., Wu, W.Z., Zhang, W.X.: Approaches to knowledge reduction based on variable precision rough set model. Information Sciences 159(3), 255-272 (2004)

6. Miao, D.Q., Zhao, Y., Yao, Y.Y., Li, H.X., Xu, F.F.: Relative reducts in consistent and inconsistent decision tables of the Pawlak rough set model. Information Sciences 179(24), 4140-4150 (2009)

7. Pawlak, Z.: Rough sets. International Journal of Computer Information and Science 11, 341-356 (1982)

8. Pawlak, Z.: Rough Sets: Theoretical Aspects of Reasoning About Data. Kluwer Academic Publishers, Dordrecht (1991)

9. Skowron, A., Rauszer, C.: The discernibility matrices and functions in information systems. In: Słowiński, R. (ed.) Intelligent Decision Support, Handbook of Applications and Advances of the Rough Sets Theory, System Theory, Knowledge Engineering and Problem Solving, vol. 11, pp. 331-362. Kluwer Academic Publishers, Dordrecht (1992)

10. Ślęzak, D.: Approximate entropy reducts. Fundamenta Informaticae 53(3-4), 365$390(2002)$

11. Zhao, Y., Yao, Y., Luo, F.: Data analysis based on discernibility and indiscernibility. Information Sciences 177(22), 4959-4976 (2007) 